\title{
Third Order Current Mode Universal Filter Using Only Op.amp. and OTAs
}

\author{
G. N. Shinde ${ }^{1}$, D. D. Mulajkar ${ }^{2}$ \\ ${ }^{1}$ Indira Gandhi (SR) College, Nanded, Maharashtra, India \\ ${ }^{2}$ Dnyanasadhana College, Thane, Maharashtra, India \\ E-mail:shindegn@yahoo.co.in \\ Received August 5, 2010; revised September 8, 2010; accepted September 15, 2010
}

\begin{abstract}
A novel current mode active-only universal filter using four dual current output Operational Transconductance Amplifiers (OTAs) and three Operational Amplifiers (OAs) is presented. The circuit can realize low pass and high pass filter characteristics by choosing the suitable current output branches. The filter performance factors natural frequency $\left(\omega_{0}\right)$, bandwidth $\left(\frac{\omega_{0}}{Q}\right)$, quality factor $\mathrm{Q}$ and transconductance gain gm are electronically tunable. The proposed circuit has very low sensitivities with respect to circuit active elements. From sensitivity analysis, it has been clearly shown that the proposed circuit has very low sensitivities with respect to the circuit active elements. The gain roll-off of high pass and low pass configuration is $18 \mathrm{~dB} / \mathrm{oc}-$ tave. The proposed circuit facilitates integrability, programmability and ease of implementation.
\end{abstract}

Keywords: Current Mode Filter, OTA, Bandwidth, Center Frequency, Circuit Merit Factor Q

\section{Introduction}

In recent years, current mode analogue signal processing circuit techniques have received wide attention due to the high accuracy, the wide signal bandwidth and the simplicity of implementing signal operations [1]. The design of current mode circuits employing active devices such as OAs, OTAs, and current conveyors (CCs) have been reported in the literature [2-6]. An OTA provides a high linear electronic tunability and wide tunable range of its transconductance gain.OTA based circuits requires no resistors; hence they are suitable for monolithic integration.

Recently, the multiple current output OTAs have been used for realizing current mode filters [7-12]. In 1996, Tsukutani et al. proposed good versatile current mode biquad filter using multiple current output OTAs and two grounded capacitors.

This paper focuses on realization of the current-mode third order active-only filter. The proposed circuit is constructed with OAs and dual current output OTAs. It is shown that the circuit can realize the biquadratic transfer function, and that the circuit characteristics can be electronically tuned by the transconductance gains of OTAs. The proposed circuit enjoys the features of:
- saving in components,

- realization of various filtering responses,

- devoid of resistors and capacitors which suits IC design techniques,

- high impedance outputs,

- electronic adjustment of $\omega_{0}$ and $\frac{\omega_{0}}{Q}$ through bias currents of the active elements

- independent electronic adjustment of passband gains,

- low sensitivity figures.

\section{Circuit Analysis and Analytical Treatment}

The open loop gain of an OA is represented by the well known first order pole model [13-15]

$$
A(S)=\frac{A_{0} \omega_{0}}{S+\omega_{0}}
$$

where $A_{0}$ Open loop D.C. gain of op-amp.

$\omega_{0}$ : Open loop $-3 \mathrm{~dB}$ bandwidth of the op-amp $=2 \pi \mathrm{f}_{0}$

$A_{0} \omega_{0}: \beta_{i}=$ gain-bandwidth product of op-amp.

For $S \gg \omega_{0}$ 


$$
A(S)=\frac{A_{0} \omega_{0}}{S}=\frac{\beta_{\mathrm{i}}}{S} \quad(\mathrm{i}=1,2,3,)
$$

This model of $\mathrm{OA}$ is valid from a few $\mathrm{kHz}$ to few hundred $\mathrm{kHz}$. In this frequency range, OTA works as an ideal device. The OTA is characterized by the port-relation

$$
\begin{aligned}
& I_{O}=g_{m}\left(V_{+}-V_{-}\right) \\
& \quad T(S)=\frac{g_{m b 0} S^{3}-\left(g_{m b 1} \beta_{1}-g_{m b 2} \beta_{2}\right) S^{2}+\left(g_{m b 2} \beta_{1} \beta_{2}-g_{m b 3} \beta_{2} \beta_{3}\right) S-g_{m b 3} \beta_{1} \beta_{2} \beta_{3}}{g_{m a 0} S^{3}+\left(g_{m a 1} \beta_{1}-g_{m a 2} \beta_{2}\right) S^{2}+\left(g_{m a 2} \beta_{1} \beta_{2}+g_{m a 3} \beta_{2} \beta_{3}\right) S+g_{m a 3} \beta_{1} \beta_{2} \beta_{3}}
\end{aligned}
$$

The circuit was designed using coefficient matching technique. i.e., by comparing these transfer functions with general third order transfer functions is given by,

$$
T(S)=\frac{\alpha_{3} S^{3}+\alpha_{2} S^{2}+\alpha_{1} S+\alpha_{0}}{S^{3}+\omega_{0}\left(1+\frac{1}{Q}\right) S^{2}+\omega_{0}^{2}\left(1+\frac{1}{Q}\right) S+\omega_{0}^{2}}
$$

Comparing Equations (1) with (2) we get,

$$
\begin{gathered}
\omega_{0}^{3}=\frac{g_{m a 3} \beta_{1} \beta_{2} \beta_{3}}{g_{m a 0}} \quad \omega_{0}^{2}\left(1+\frac{1}{Q}\right)=\frac{g_{m a 1} \beta_{1}+g_{m a 2} \beta_{2}}{g_{m a 0}} \\
\omega_{0}\left(1+\frac{1}{Q}\right)=\frac{g_{m b 2} \beta_{1} \beta_{2}+g_{m b 3} \beta_{2} \beta_{3}}{g_{m a 0}} \\
\alpha_{3}=\frac{g_{m b 0}}{g_{m a 0}} \text { And } \alpha_{0}=\frac{g_{m b 3}}{g_{m a 0}}
\end{gathered}
$$

It is found from above equations that circuit parameters $\omega_{0}, Q ; \alpha_{0}$ can independently set and electronically where, $g_{m}$ is transconductance of OTA. In the dual current output OTA, the plus current output has a positive polarity, and the minus current output has a negative polarity.

The analysis gives the current transfer function $T=[I$ out / I in] as follows:

$$
\begin{aligned}
& T_{H P}=\frac{\alpha_{0} S^{3}}{g_{m a 0} S^{3}+\left(g_{m a 1} \beta_{1}+g_{m a 2} \beta_{2}\right) S^{2}+\left(g_{m a 2} \beta_{1} \beta_{2}+g_{m a 3} \beta_{2} \beta_{3}\right) S+g_{m a 3} \beta_{1} \beta_{2} \beta_{3}} \\
& T_{L P}=\frac{\alpha_{3}}{g_{m a 0} S^{3}+\left(g_{m a 1} \beta_{1}+g_{m a 2} \beta_{2}\right) S^{2}+\left(g_{m a 2} \beta_{1} \beta_{2}+g_{m a 3} \beta_{2} \beta_{3}\right) S+g_{m a 3} \beta_{1} \beta_{2} \beta_{3}}
\end{aligned}
$$

The realization of the other transfer functions invariably requires matching the conditions in terms of the transconductance gains of the OTAs and the gain-bandwidth products of the OAs.

The transconductance gains of the OTAs to realize the desired characteristics are obtained from (3) as

$$
\begin{gathered}
g_{m a 3}=\frac{\omega_{0}^{3} \beta_{1} \beta_{2} \beta_{3}}{g_{m a 0}} \\
g_{m a 2}=\frac{\omega_{0}^{2}}{\beta_{1} \beta_{2}}\left\{\left(1+\frac{1}{Q}\right)-\frac{g_{m a 0} \omega_{0}}{\beta_{1} \beta_{2} \beta_{3}}\right\} \\
g_{m a 1}=\frac{g_{m a 0} \omega_{0}}{\beta_{1}}\left(1+\frac{1}{Q}\right)-\frac{g_{m a 2} \beta_{2}}{\beta_{1}}
\end{gathered}
$$

where $\omega_{0}, Q, \beta_{1}, \beta_{2}, \beta_{3}$ and $g_{m a 0}$ should be given in advance. tuned adjusting the transconductance gains of the OTAs. If $g_{m a}, \beta_{1}, \beta_{2}$ and $\beta_{3}$ are given, the parameter $\omega_{0}$ can be set by $g_{m a 2}$. The parameters $Q$ and $\alpha_{3}$ can be set by $g_{m a 1}$ and $g_{m b 0}$ respectively. It seems that the values of $Q$ and $\alpha_{3}$ are also limited by the dynamic ranges of the OA and OTA.

From (1), it can be seen that:

1) The low pass transfer function can be realized with $g_{m b 0}=0$

$$
g_{m b 1} \beta_{1}=g_{m b 2} \beta_{2} \text { and } g_{m b 2} \beta_{1} \beta_{2}=g_{m b 3} \beta_{2} \beta_{3}
$$

2) The high pass transfer function can be realized with $g_{m b 3}=0 \quad g_{m b 1} \beta_{1}=g_{m b 2} \beta_{2}$ and $g_{m b 2} \beta_{1} \beta_{2}=g_{m b 3} \beta_{2} \beta_{3}$

3) The band pass transfer function can be realized with $g_{m b 3}=g_{m b 0}=0 \quad g_{m b 1} \beta_{1}=g_{m b 2} \beta_{2}$

The high pass and low pass transfer functions obtained are as follows,
Methods of implementing a dual current output OTA have been discussed previously (Ramirez-Angulo et al. 1992, Wu 1994).

\section{Circuit Diagram}

The diagram was shown in Figure 1.

\section{Circuit Description}

The proposed circuit is built with four dual current output OTAs and three OAs is as shown in Figure (1). The $\mathrm{V}+$ terminal of first OTA and V-terminal of all other OTAs are grounded. Output terminal of first OTA carrying positive polarity current is fed to inverting terminal of first OA. Its output is fed to inverting terminal of 


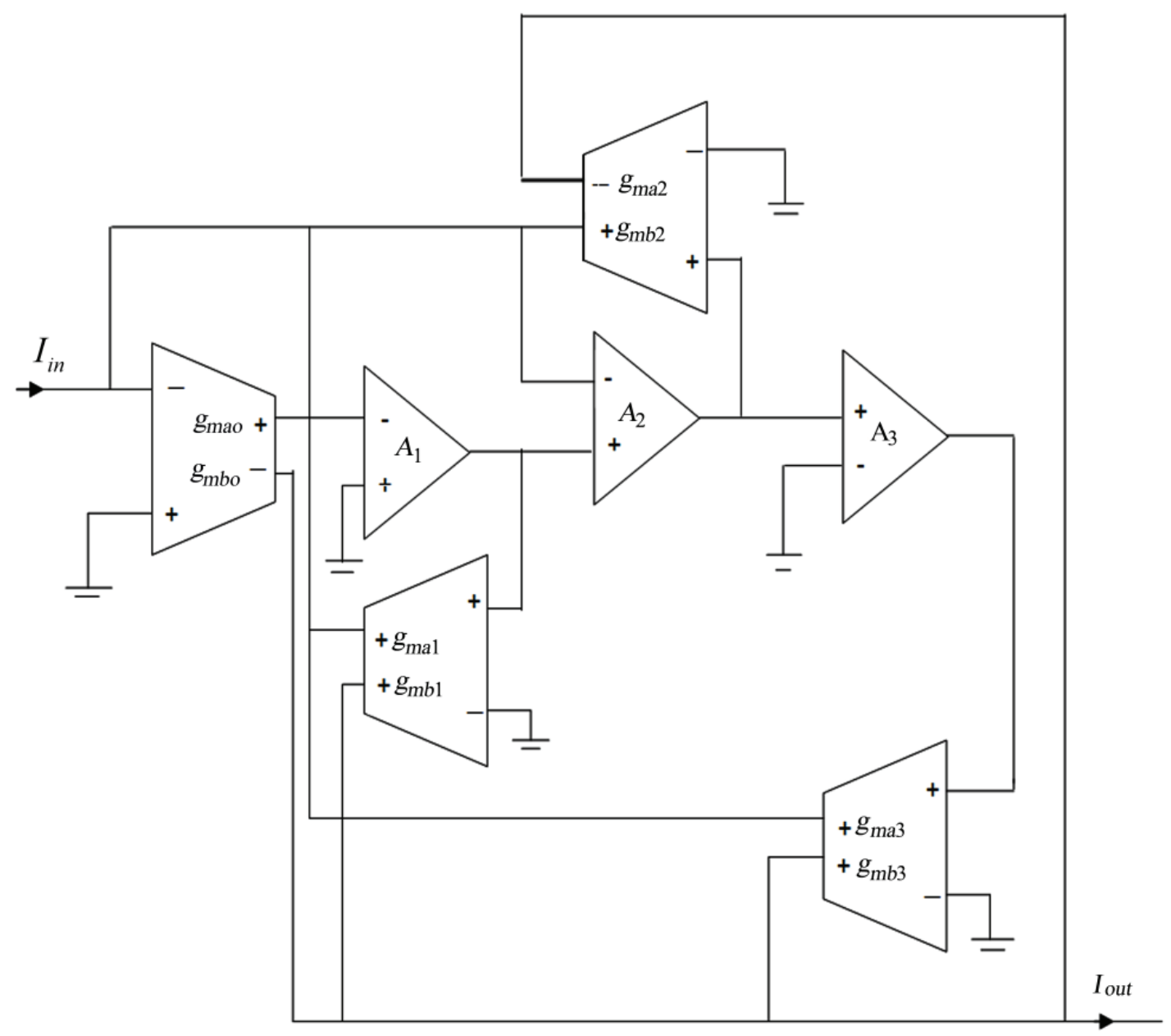

Figure 1. Circuit diagram of electronically tunable third order current-mode universal filter.

non-inverting of third OA output of third OA is then fed to $\mathrm{v}+$ terminal fourth OTA. Output terminals of all OTAs carrying positive current are fed to inverting of first OA whereas remaining current output terminals of all OTAs adds to give output current of the circuit. The circuit can realize various third order filter functions by suitably choosing the current output branches.

\section{Result and Discussion}

The circuit performance is studied for Central frequencies $f_{0}=100 \mathrm{kHz}$ and $1 \mathrm{MHz}$ with circuit merit factor Q $=1$. The general operating range of this filter is $10 \mathrm{~Hz}$ to $1 \mathrm{MHz}$. The value of $\beta_{1}=\beta_{2}=\beta_{3}=6.392 \times 10^{6}$ for LF $356 \mathrm{~N}$. The proposed circuit gives response only for very high frequencies since the values of transconductance of OTAs takes very low values at frequencies less than 100 $\mathrm{kHz}$. The values of $g_{m a 1}, g_{m a 2}$ and $g_{m a 3}$ are calculated by taking $g_{m a 0}=2$ and $\frac{g_{m a 3}}{g_{m a 0}}=1$ Response is studied for
$\mathrm{Q}=1$ for high pass and low pass function. Figures 2 and 3 shows high pass and low pass response of the proposed filter circuit respectively. Data obtained after analysis high pass and low pass response is given in Tables 2 and 3. From Figures $\mathbf{2}$ and $\mathbf{3}$, it is seen that the gain roll-off is $18 \mathrm{~dB} /$ octave for both the functions and the gain stabilizes to $0 \mathrm{~dB}$ at frequency $200 \mathrm{~Hz}$. There is no overshoot in the response. Observed $-3 \mathrm{~dB}$ frequency i.e. cutoff frequency matches with designed value $f_{0}$. Thus the filter circuit works ideal for high pass as well as low pass function. The values of transconductance gains for $f_{0}=$ $100 \mathrm{kHz}$ and $1 \mathrm{MHz}$ obtained are given in Tables 1(a) and (b) respectively.

\section{Sensitivities}

The practical solution is to design a network that has low sensitivity to element changes $[14,15]$. Thus sensitivity must be less than limit i.e. unity. The lower the sensitivity of the circuit, the less will its performance deviate because of element changes. The sensitivities $S_{x}^{\omega_{0}}$ and 
$S_{x}^{\alpha_{3}}$ with respect to the circuit active elements are shown in Table 4. These values are within the range $0 \leq S_{x}^{y} \leq 1$ It is found that the proposed circuit has very low sensitivity with respective to active elements.

\section{Concluding Remarks}

A versatile current-mode active-only filter using OAs and OTAs has been proposed. The proposed circuit can

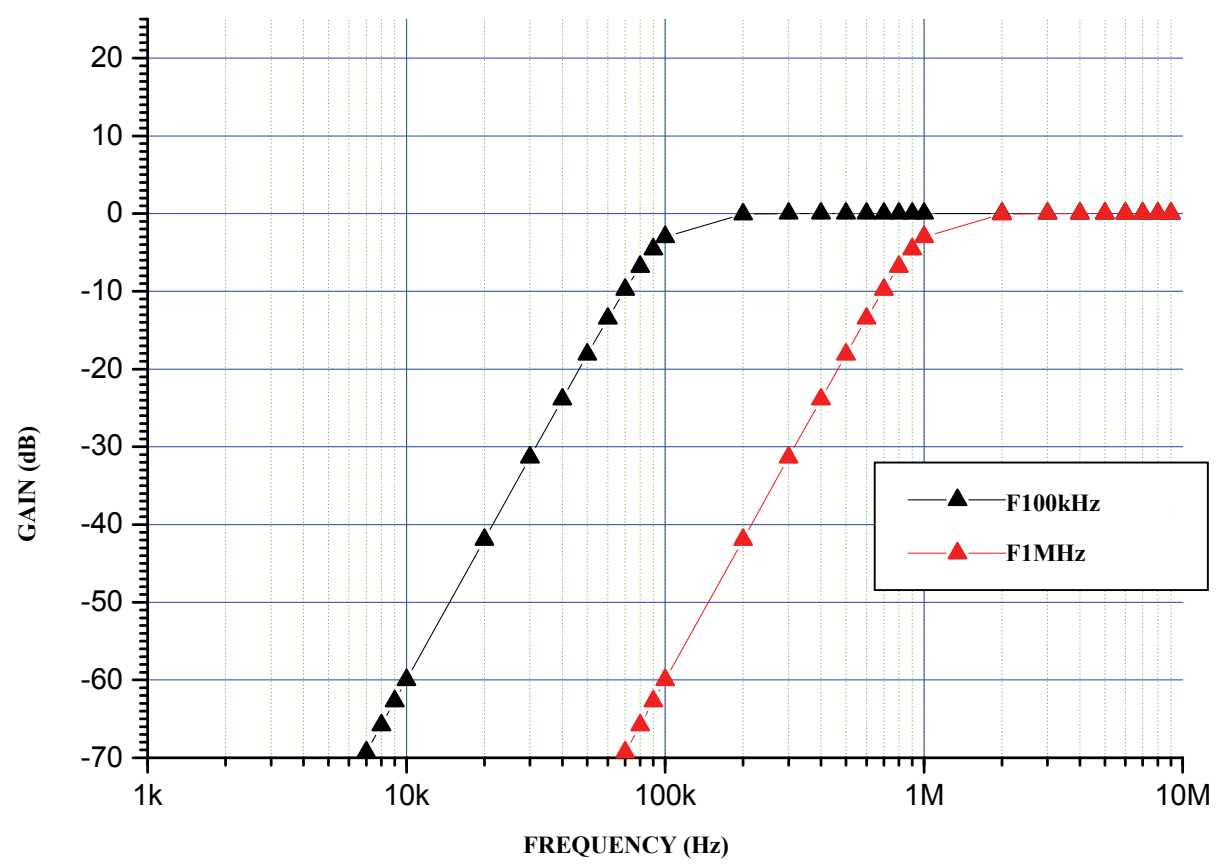

Figure 2. High pass response of proposed current-mode filter.

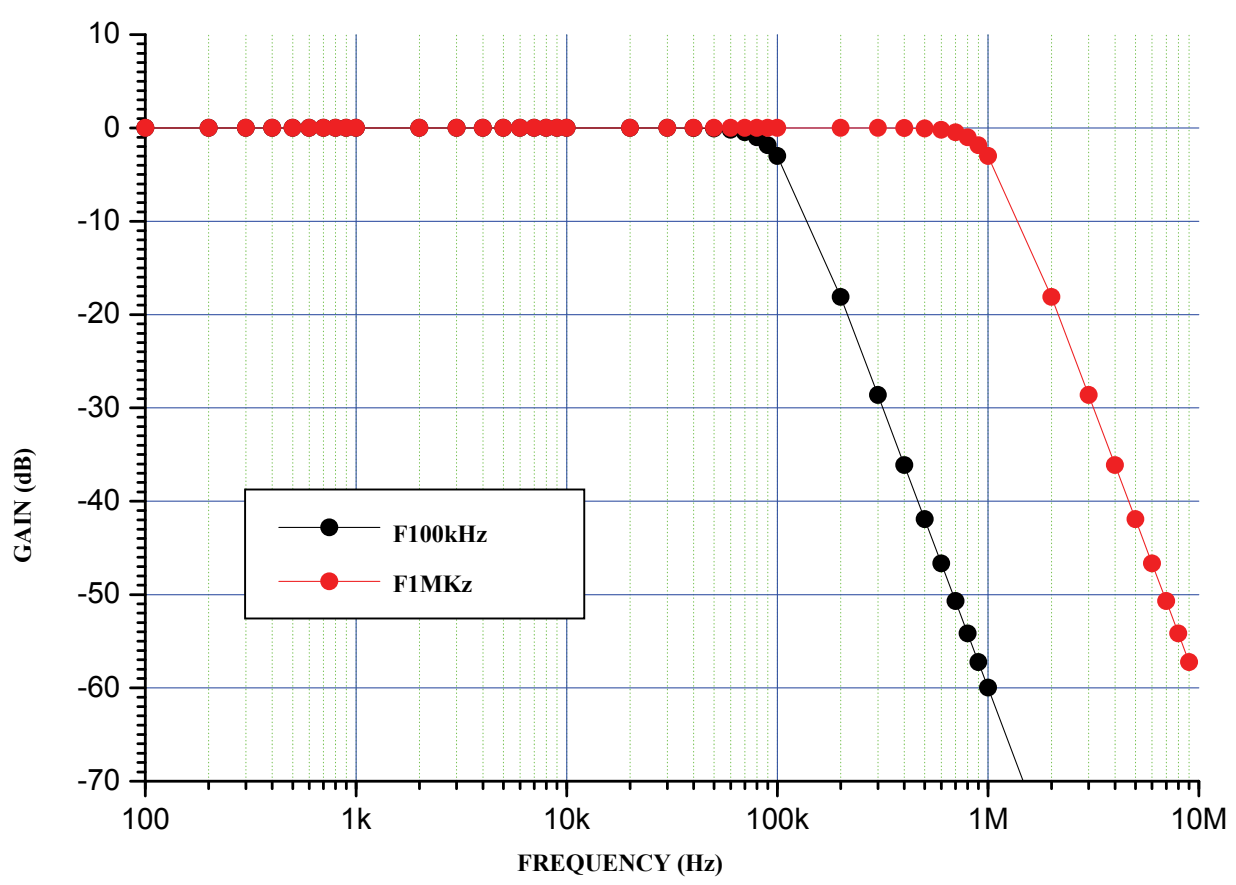

Figure 3. Low pass response of proposed current-mode filter. 
Table 1. The values of transconductance gains.

\begin{tabular}{cc}
\hline$g_{m a}$ & Value in $\mathrm{mS}$ for $f_{0}=100 \mathrm{kHz}$ \\
\hline$g_{m a 0}$ & 2 \\
$g_{m a 1}$ & 0.356 \\
$g_{m a 2}$ & 0.0367 \\
$g_{m a 3}$ & 0.0019 \\
$g_{m b 0}$ & 2 \\
$g_{m b 3}$ & 0.0019 \\
\hline
\end{tabular}

(a) for $f_{0}=100 \mathrm{kHz}$

\begin{tabular}{cc}
\hline$g_{m a}$ & Value in $\mathrm{mS}$ for $f_{0}=\mathrm{MHz}$ \\
\hline$g_{m a 0}$ & 2 \\
$g_{m a 1}$ & 1.966 \\
$g_{m a 2}$ & 1.965 \\
$g_{m a 3}$ & 1.9 \\
$g_{m b 0}$ & 2 \\
$g_{m b 3}$ & 1.9 \\
\hline
\end{tabular}

(b) for $f_{0}=1 \mathrm{MHz}$

Table 2. Analysis of frequency response of high pass function for $Q=1$.

\begin{tabular}{|c|c|c|c|c|c|c|}
\hline \multirow{2}{*}{$\begin{array}{c}f_{0} \\
(\mathrm{kHz})\end{array}$} & \multirow{2}{*}{$\begin{array}{c}F_{O H} \\
(\mathrm{kHz})\end{array}$} & \multirow{2}{*}{$\begin{array}{l}f_{0} \sim F_{O H} \\
(\mathrm{kHz})\end{array}$} & \multicolumn{2}{|c|}{$\begin{array}{l}\text { Gain Roll-off in } \\
\text { stop band }\end{array}$} & \multicolumn{2}{|c|}{$\begin{array}{l}\text { Gain Stabi- } \\
\text { lization }\end{array}$} \\
\hline & & & $\mathrm{dB} /$ Octave & $\begin{array}{c}\text { Octave } \\
\text { starting at } \\
(\mathrm{kHz})\end{array}$ & $\mathrm{dB}$ & $\begin{array}{r}F_{S} \\
(\mathrm{kHz})\end{array}$ \\
\hline 100 & 100 & 0 & 18 & 500 & 0 & \\
\hline $1 \mathrm{M}$ & $1 \mathrm{M}$ & 0 & 18 & 500 & 0 & $2 \mathrm{M}$ \\
\hline \multicolumn{3}{|c|}{$F_{O H}:-3 \mathrm{~dB}$ Frequency } & \multicolumn{4}{|c|}{$F_{S}:$ Frequency at which gain stabilizes } \\
\hline
\end{tabular}

Table 3. Analysis of frequency response of low pass function for $Q=1$.

\begin{tabular}{|c|c|c|c|c|c|c|}
\hline \multirow{2}{*}{$\begin{array}{c}f_{0} \\
(\mathrm{kHz})\end{array}$} & \multirow{2}{*}{$\begin{array}{r}F_{O L} \\
(\mathrm{kHz})\end{array}$} & \multirow{2}{*}{$\begin{array}{c}f_{0} \sim F_{O L} \\
(\mathrm{kHz})\end{array}$} & \multicolumn{2}{|c|}{$\begin{array}{l}\text { Gain Roll-off in } \\
\text { stopband }\end{array}$} & \multicolumn{2}{|c|}{$\begin{array}{l}\text { Gain Stabili- } \\
\text { zation }\end{array}$} \\
\hline & & & $\mathrm{dB} /$ Octave & $\begin{array}{c}\text { Octave } \\
\text { starting } \\
\text { at } \\
(\mathrm{kHz})\end{array}$ & $\mathrm{dB}$ & $\begin{array}{c}F_{S} \\
(\mathrm{~Hz})\end{array}$ \\
\hline 100 & 100 & 0 & 18 & 400 & 0 & 100 \\
\hline $1 \mathrm{M}$ & $1 \mathrm{M}$ & 0 & 18.3 & $2 \mathrm{M}$ & 0 & 100 \\
\hline \multicolumn{7}{|c|}{$\mathrm{F}_{\mathrm{OL}}:-3 \mathrm{~dB}$ Frequency } \\
\hline
\end{tabular}

realize the biquadratic transfer function and the circuit characteristics can be electronically tuned by the transconductance gains. From sensitivity analysis, it has been clearly shown that the proposed circuit has very low sensitivities with respect to the circuit active elements.
Table 4. Sensitivities $S_{x}^{\omega_{0}}$ and $S_{x}^{\alpha_{3}}$.

\begin{tabular}{ccc}
\hline$x$ & $S_{x}^{\omega_{0}}$ & $S_{x}^{\alpha_{3}}$ \\
\hline$g_{m a 0}$ & -0.33 & -1.0 \\
$g_{m a 1}$ & 0 & 0 \\
$g_{m a 2}$ & 0 & 0 \\
$g_{m a 3}$ & 0.33 & 0 \\
$g_{m b 0}$ & 0.33 & 1.0 \\
$\beta_{1}$ & 0.33 & 0 \\
$\beta_{2}$ & 0.33 & 0 \\
$\beta_{3}$ & 0.33 & 0 \\
\hline
\end{tabular}

The gain roll-off of high pass and low pass configuration is $18 \mathrm{~dB} /$ octave.

\section{References}

[1] T. Tsutani, T. Higashimura, Y. Sumi and Y. Fukui, "Electonically Tunable Current Mode Active Only Biquadratic Filter," International Journal of Electronics, Vol. 87, No. 3, 2000, pp. 307-314.

[2] C.-C. Hsu and W.-S. Feng, "Dynamic Decoupling and Compensating Methods of Multi-Axis Force Sensors," International Journal of Electronics, Vol. 88, No. 1, 2001, pp. 41-51.

[3] I. A. Khan and M. H. Zaidi, "Multifunction Translinear-C Current - Mode Filter," International Journal of Electonics, Vol. 87, No. 9, 2000, pp. 1047-1051.

[4] M. Higashimura, "Current Mode Low Pass and Band Pass Filters Using the Operational Amplifier Pole," International Journal of Electronics, Vol. 74, No. 6, 1993, pp. 945-949.

[5] T. M. Ishida, Y. Fukui and S. Tsuiki, "Novel Electronically Tunable Current Mode Filter without External Passive Elements," IEEE, Vol. 1, 1996, pp. 262-265.

[6] G. W. Roberts and A. S. Sedra, "A General Class of Current Amplifier Based Biquadratic Filter Circuits," IEEE Transactions on Circuits and Systems, Vol. 39, No. 4, 1992, pp. 257-263.

[7] J. Ramirez-Angulo, M. Robinson and E. Sanchez-Sinencio, "Current Mode Continuous Time Filters Two Design Approaches," IEEE Transactions on Circuits and Systems, Vol. 39, No. 6, 1992, pp. 337-341.

[8] N. A. Shah, M. F. Rather, M. A. Malik and S. Z. Iqbal, "Cascadable Electronically Tunable Sito Current Mode Active Only Universal Filter," ETRI Journal, Vol. 26, 2004, pp. 295-300.

[9] A. K. Mitra and V. K. Aatre, "Low Sensitivity HighFrequency Active R Filters," I.E.E.E. Transactions on Circuits and Systems, Vol. 23, No. 11, 1976, pp. 670676.

[10] G. N. Shinde and P. B. Patil, "Sadhana," Journal of Engineering Science, Vol. 28, No. 6, 2003, pp. 1919-1926.

[11] T. Tsukutani, M. Higashimura, Y. Sumi and Y. Fukui, "Novel Voltage-Mode Biquad Using Only Active Devic- 
es," International Journal of Electronics, Vol. 187, No. 3, 2000, pp. 307-314.

[12] R. Nandi "Active R Realization of Bilinear RL Impedances and their Applications in a High-Q Parallel Resonator and External Oscillator," Proceeding of the Institute of Electrical and Electronics Engineering, Vol. 66, No. 12, 1978, pp. 1666-1668.

[13] G. N. Shinde and D. D. Mulajkar, "Electronically Tunable Current-Mode Second Order High Pass Filter for Different Value of Q," International Journal of Physical
Sciences, Vol. 3, No. 6, 2008, pp. 148-151.

[14] D. R. Bhaskar, U. R. Sharma and S. M. I. Rizvi, "New Current-Mode Universal Biquadratic Filter," Microelectronic Journal, Vol. 88, No. 10, 1999, pp. 837-839.

[15] S. Minaei and S. Turkoz, "Current-Mode Electronically Tunable Universal Filter Using Only Plus-Type Current Controlled Conveyors and Grounded Capacitors, Bandpass High Pass Filter," ETRL Journal, Vol. 26, No. 4, 2004, pp. 292-296. 